\title{
Archéopages
}

Archéopages

Archéologie et société

40 | 04-07/2014

Villages

\section{Le village évanescent. Formes de l'habitat à l'âge du Bronze en France méditerranéenne}

\section{Thibault Lachenal}

\section{(2) OpenEdition}

12 Journals

Édition électronique

URL : https://journals.openedition.org/archeopages/590

DOI : 10.4000/archeopages.590

ISSN : 2269-9872

\section{Éditeur}

INRAP - Institut national de recherches archéologiques préventives

\section{Édition imprimée}

Date de publication : 1 juin 2015

Pagination : 26-35

ISSN : $1622-8545$

\section{Référence électronique}

Thibault Lachenal, « Le village évanescent. Formes de I'habitat à l'âge du Bronze en France

méditerranéenne », Archéopages [En ligne], 40 | 04-07/2014, mis en ligne le 01 juillet 2016, consulté le 22 janvier 2022. URL : http://journals.openedition.org/archeopages/590 ; DOI : https://doi.org/

10.4000/archeopages.590 


\section{Le village évanescent \\ Formes de l'habitat à lâge du Bronze en France méditerranéenne}

Thibault Lachenal CNRS, UMR 5140 «Archéologie des sociétés méditerranéennes»"

\section{6}

\section{Dégagé sur une \\ cinquantaine de mètres \\ de longueur, le rempart du \\ Camp de Laure, qui devait \\ atteindre les $2 \mathrm{~m}$ de \\ hauteur, est construit à \\ double parement en blocs \\ grossièrement \\ appareillés. À l'extérieur, \\ un mur délimite un espace \\ interprété comme un \\ " chemin de ronde ". \\ L'entrée est encadrée de \\ deux bastions et l'on \\ retrouve, adossés au mur \\ externe, des amas de \\ pierres, équidistants \\ d'environ $25 \mathrm{~m}$, \\ correspondant à de \\ possibles tours.}
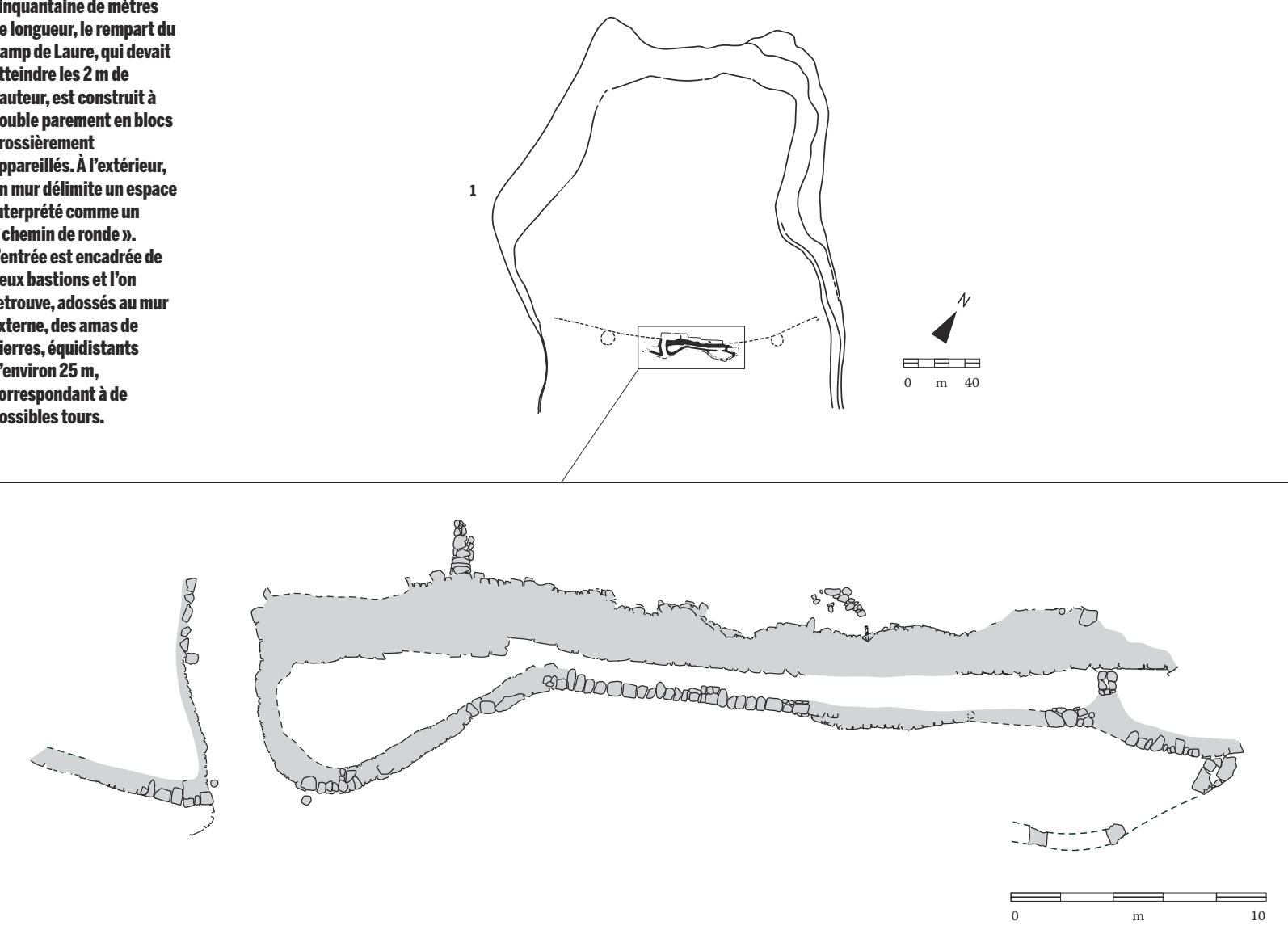
Traiter du village à l'âge du Bronze en France, $a$ fortiori dans le Midi, relève de la gageure. En effet, si le terme est aujourd'hui largement employé dans les travaux des protohistoriens, en particulier pour ce qui concerne l'habitat aggloméré de l'âge du Fer, son acception rencontre une résistance certaine chez les spécialistes de l'âge du Bronze. Une synthèse récente (Carozza, Marcigny, 2007, p. 61), ainsi que les contributions à un séminaire du Collège de France abordant ce thème (Mordant, 2008, p. 213 ; Vital, 2008, p. 186), illustre bien cette réticence. Son emploi est réservé à des cas bien particuliers, faisant figure d'exception dans le paysage rural de l'âge du Bronze ouest-européen. Le vocable de hameau, lui, est bien souvent préféré pour désigner les habitats groupés de petite dimension, dont les découvertes sont plus fréquentes. Cette banalisation de la notion de village observable par ailleurs venait pourtant de la volonté de simplifier le terme (Audouze, Buchsenschutz, 1989), en regard de la définition, trop précise pour être appliquée à la Protohistoire, qu'en donnent les spécialistes du Moyen Âge, laquelle est d'ailleurs à la source de débats épistémologiques décennaux au sein de cette discipline (Watteaux, 2003). Il était ainsi proposé de l'étendre à « toutes les agglomérations réunissant plusieurs habitations, les termes de ferme ou de hameau étant réservés aux groupements de deux ou trois bâtiments, sans référence à leur fonction » (Audouze et Buchsenschutz, 1989, p. 234). Cette conception n'est pas en soi incompatible avec les définitions lexicale et géographique du village qui restent assez ouvertes et se rejoignent sur le concept d'agglomération rurale. Aucun seuil de population ou de surface ne sont précisés, lesquels sont d'ailleurs fluctuants dans le temps et selon les territoires. En revanche, le terme de hameau semble pour sa part plus connoté, puisque la notion de subordination à une agglomération plus importante (le village) revient très fréquemment dans ses définitions.

La France méditerranéenne n'est probablement pas le terrain privilégié pour apporter des réponses à ces questionnements. Les recherches programmées sur les sites de plein air ont été pendant longtemps délaissées au profit des occupations en abri naturel. Le développement de l'archéologie préventive a sans nul doute renversé les codes. Le diagnostic des zones de plaines alluviales où les vestiges ne sont pas décelables en surface a permis de mettre en évidence d'autres aspects des habitats des sociétés de l'âge du Bronze. Néanmoins, cet apport n'y a pas été aussi intense que dans d'autres régions. La prise en compte croissante des spécificités de cette période dans les stratégies de prescription influe sur la nature et la quantité des sites fouillés et laisse présager une multiplication des découvertes dans les années qui viennent plutôt qu'une stabilisation des connaissances. Notre objectif est donc de dresser un bilan de la documentation disponible en France méditerranéenne, afin d'en extraire des conclusions et des hypothèses sur l'évolution de l'organisation des sites domestiques.

\section{Les sites perchés et fortifiés du début de l'âge du Bronze}

L'un des habitats emblématiques de l'âge du Bronze ancien du midi de la France, site éponyme du style à décor barbelé caractérisant les premières productions céramiques de cette période (Vital et al., 2012), est le Camp de Laure au Rove, dans les Bouches-du-Rhône. Il s'agit d'un éperon calcaire d'une superficie de plus de 1,5 ha limité au nord par des abrupts calcaires et fermé au sud par une levée de pierres qui coupe le plateau sur $145 \mathrm{~m}$ [ill. 1]. Celle-ci résulte de l'effondrement d'un système de fortification sur lequel se sont focalisées les fouilles archéologiques (Courtin, 1975 ; Vital et al., 2012). Le matériel, qui a été découvert principalement à l'intérieur, le long du rempart, évoque clairement une occupation domestique. Si le reste du plateau n'a pas été exploré, c'est essentiellement car ce dernier est très érodé et que les niveaux de sols archéologiques n'ont pas été conservés. Il reste toutefois plausible que cet ouvrage défensif ait enclos un habitat plus vaste. Ce dernier ne connaît pas d'équivalent direct en France méditerranéenne. Le site du Clos Marie-Louise à Aix-en-Provence (Bouches-du-Rhône) pourrait constituer un élément de comparaison. Bien que de taille plus modeste (o,2 ha), cet éperon est fermé au nord-ouest par une levée de terre et de pierres qui le barre sur près de $30 \mathrm{~m}$ de long. La datation de cette dernière n'est pas assurée, mais le site a notamment livré un mobilier très comparable à celui du Camp de Laure (Vignaud, 2002), attribuable au Bronze ancien 1 (BA 1, 21001950 avant notre ère). Cet attrait pour les sites de hauteur est confirmé par les découvertes réalisées au Baou-Roux à Bouc-Bel-Air. Les formes de l'habitat du début du Bronze ancien n'y sont toutefois pas connues, ni son extension qui, selon Philippe Boissinot, intéressait toutefois la totalité du plateau (Boissinot, 1993). La concentration de découvertes de cette période sur des sites perchés localisés sur les pourtours de l'étang de Berre (Miouvin à Istres, Saint-Blaise à Saint-Mitre-lèsRemparts, Saint-Pierre-les-Martigues, le Collet Redon à La Couronne, le Baou Majour à Grans) est également remarquable. Elle renforce le caractère exceptionnel des sites fortifiés du Camp de Laure et éventuellement du Clos Marie-Louise, lui aussi localisé à proximité. Ces derniers ont pu jouer un rôle structurant dans un territoire constitué d'habitats dispersés de plus petite dimension. Cette expérience ne semble pas avoir été portée plus avant, elle paraît correspondre à un épiphénomène qui ne retrouvera des éléments de comparaison que vers la fin de l'âge du Bronze. L'origine pourrait en être recherchée dans les 


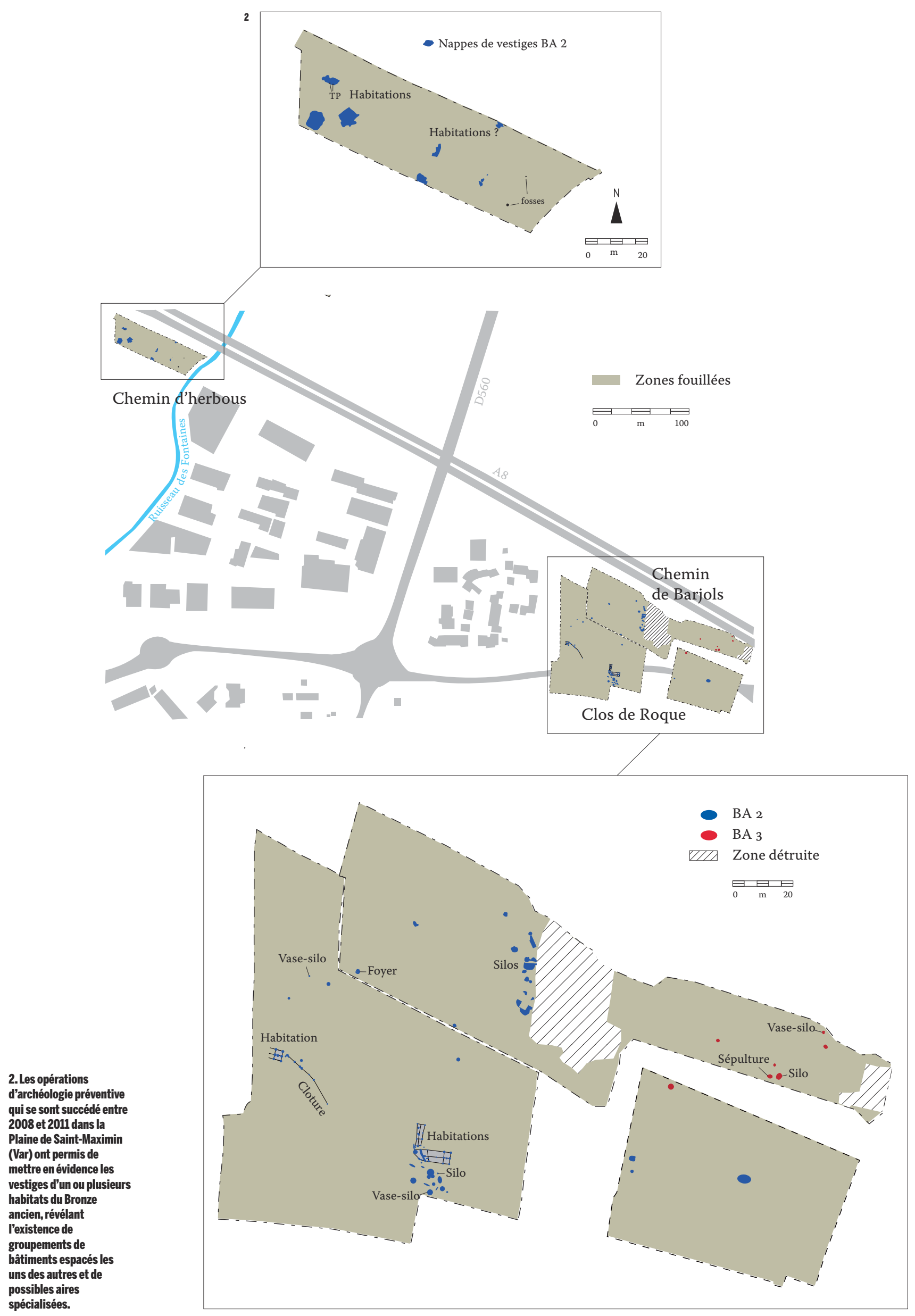


éperons barrés de la fin du Néolithique tels que les Lauzières à Lourmarin (Vaucluse) ou le Rocher du Causse à Claret (Hérault) qui ont également fait l'objet de réoccupations au Bronze ancien (D’Anna et al., 1989 ; Coularou et al., 2004).

Les agglomérations de plaine du Bronze ancien Les établissements de plaine sont connus dès la phase ancienne du Bronze ancien, en particulier dans le département du Vaucluse aux Petites Bâties à Lamotte du Rhône (Vital et al., 2012) et aux Juilléras à Mondragon (Lemercier, 2002). Sur ce site occupé au Néolithique et au Bronze ancien 1, plusieurs structures domestiques, ainsi qu'une petite nécropole rassemblant neuf tombes, peuvent être rattachées à cette dernière phase. Une partition fonctionnelle de l'espace peut être décelée dans l'organisation des aménagements avec, au nord, des fosses de rejets, au centre des foyers, et au sud, des creusements remplis de pierres interprétés comme des structures de maintien d'une palissade, tandis que le groupe funéraire se développe sur le côté est de la fouille (Lemercier, 2002, fig. 6). Bien qu'aucun plan de bâtiment ne soit connu, l'association entre un espace domestique et une petite nécropole plaide en faveur d'un établissement relativement pérenne. En effet, cette configuration ne se retrouve au Bronze ancien que sur des sites de très grande extension pour lesquels le statut de village ne laisse que peu de doute, tels que Machal à Dallet (Loison, 2003) ou Petit Beaulieu à Clermont-Ferrand dans le Puy-de-Dôme (Thirault et al., 2013).

Pour la phase moyenne du Bronze ancien (BA 2), qui se développe entre 1950 et 1850 avant notre ère (Lachenal, 2014a), les données proviennent essentiellement de la plaine de SaintMaximin dans le Var [ill. 2]. À Chemin d'Herbous, l'occupation du Bronze ancien est caractérisée par des nappes de mobilier de forme quasi rectangulaire, dont l'une est bordée de deux trous de poteaux (Donnelly, Furestier, 2009). Ces vestiges correspondraient selon nous à trois à cinq cellules d'habitation, dont les mieux conservées permettent de restituer des espaces internes d'environ $25 \mathrm{~m}^{2}$. À près de $500 \mathrm{~m}$ plus à l'est, deux opérations réalisées aux lieudits Chemin de Barjols (Cockin, Furestier, 2009) et Clos de Roque (Remicourt et al., 2014) ont permis de dégager des vestiges du Bronze ancien répartis sur une surface de près de 2 ha, parmi lesquels une distribution fonctionnelle des aménagements est décelable. Ainsi, les témoins interprétables comme des structures d'habitats sont localisés au sud-ouest du site, sur la parcelle du Clos de Roque. Un premier groupe de deux bâtiments d'orientations perpendiculaires, mesurant respectivement 13 et $38 \mathrm{~m}^{2}$, sont associés au sud à des rangées de trous de piquets, à un silo de petite dimension et aux restes d'un vase de stockage semi-enterré. Plus à l'ouest, un autre bâtiment, probablement à architecture mixte (poteaux plantés et sablière basse), est localisé près d'une clôture d'extension limitée. Dans la partie nord du site, un groupe d'au moins sept fosses de grandes dimensions, associées à d'autres structures plus superficielles, peuvent être interprétées comme un groupement de silos. D'autres vestiges disséminés sur l'emprise de la fouille peuvent signaler l'existence d'autres habitations, dégradées ou hors emprise.

L'organisation du site de Chemin de Barjols/ Clos de Roque trouve des parallèles avec le schéma mis en évidence en moyenne vallée du Rhône à la même période, avec le regroupement de constructions de surface réduites, à vocation familiale, dont une certaine autonomie est garantie par la présence de clôtures (Vital, 2008). La notion d'agglomération est toutefois ici moins flagrante. Si deux bâtiments sont très proches (mais il pourrait tout aussi bien s'agir d'un habitat et d'une dépendance), le troisième s'en écarte notablement. Une autre différence majeure se remarque dans la répartition des structures de stockage. Bien que certains aménagements de faible volume (silo de petite dimension et vase enterré) puissent être associés à l'utilisation exclusive d'une maisonnée, la plupart des grandes fosses se regroupent dans la partie nord du site, dans une zone qui a malheureusement été en grande partie endommagée. Ce regroupement des structures de conservation des produits agricoles en marge de l'habitat va dans le sens d'une gestion collective de cette activité. Le choix de recourir à une organisation communautaire, de manière à dégager un surplus, pourrait être le moteur de ce phénomène d'agglomération, ainsi que Joël Vital l'a proposé en moyenne vallée du Rhône (ibid., p. 186), selon des modalités différentes toutefois. Les découvertes de Chemin d'Herbous incitent également à élargir notre focale d'analyse. Ces habitations distantes de $500 \mathrm{~m}$, qui ne sont pas associées à des structures de conservation, pourraient être intégrées dans le même système communautaire, selon une forme d'agglomération rurale à maille très lâche. Il faut noter que les traditions architecturales des bâtiments, tant dans la forme que dans les techniques mises en œuvre, sont très diverses, ce qui renvoie une fois de plus à ce qui a pu être observé en vallée du Rhône (ibid., p. 186).

Au nord-est de la fouille de Chemin de Barjols se concentrent des structures attribuées à une phase postérieure du Bronze ancien 3 ( $\left.\mathrm{BA}_{3}\right)$, vers 1850-1650/1600 avant notre ère (Lachenal, 2014a), suggérant un déplacement de l'habitat dans cette direction. Leur plus faible nombre ne permet pas de commenter l'organisation des structures. Notons toutefois la présence d'une sépulture en fosse circulaire, d'un silo et d'un probable vase de stockage enterré. S'il est envisageable que le site se prolonge en dehors de la zone investiguée, il semble néanmoins présenter une extension plus restreinte que l'occupation précédente. 


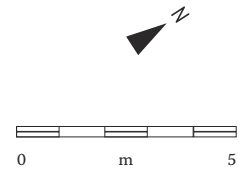

Fosses du Bronze ancien

Grandes structures du Bronze ancien

Fosses du Bronze moyen

Fosses repérées en surface
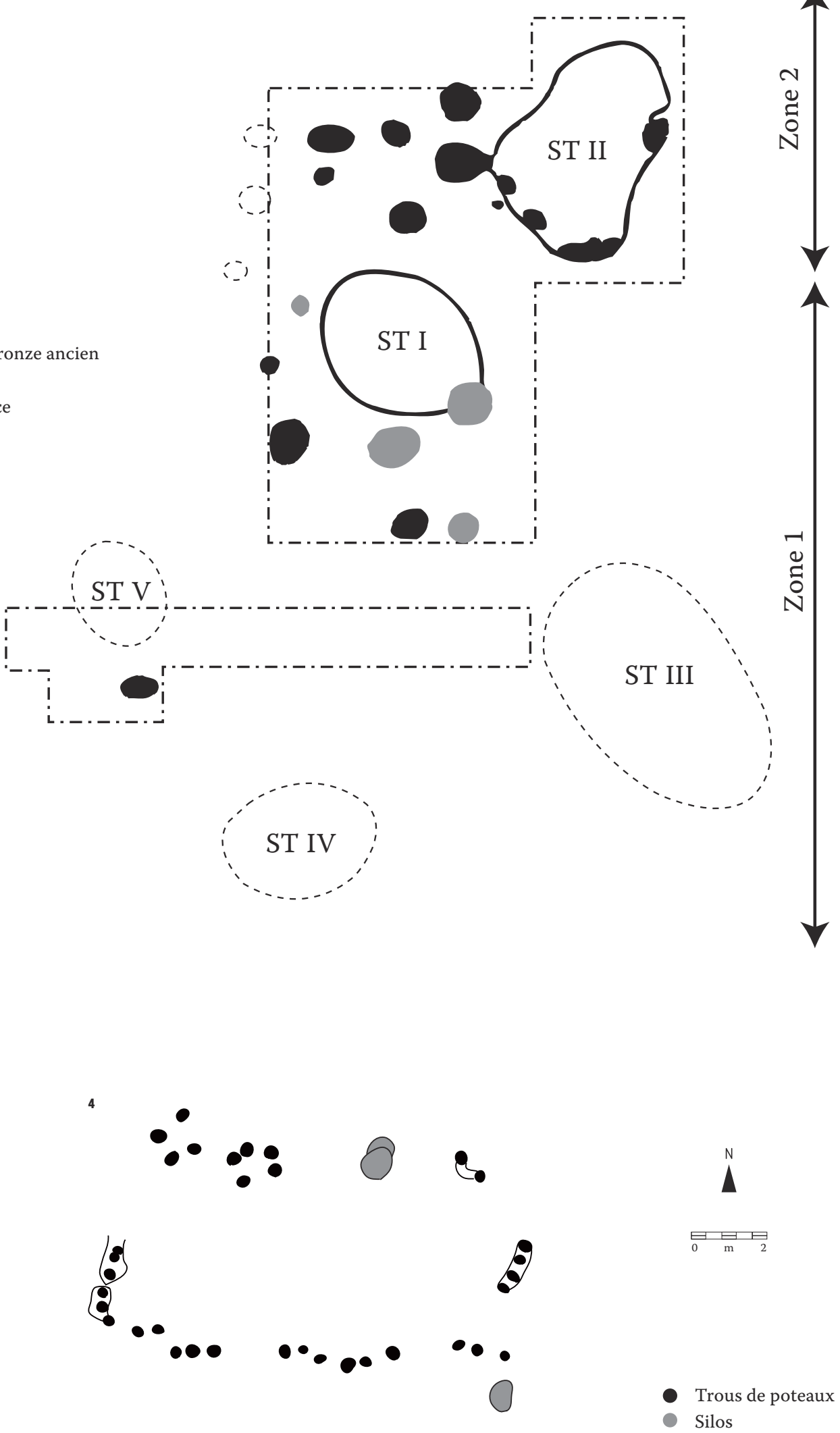

Perrier à Codognan

(Gard), aucun plan de

bâtiment n'a pu être

observé, les cinq grandes

structures excavées

identifiées à la fouille

devant plutôt se rapporter

à des creusements pour

l'extraction de sédiments

(Vital, 2004) ou à des

groupes de fosses

perturbées par les labours.

4. Le site du Moullard sud

à Lambesc (Bouches-du-

Rhône) a livré un bâtiment

isolé d'une cinquantaine

de mètres carrés attribué

au Bronze moyen, auquel

sont associées des

structures de stockage.

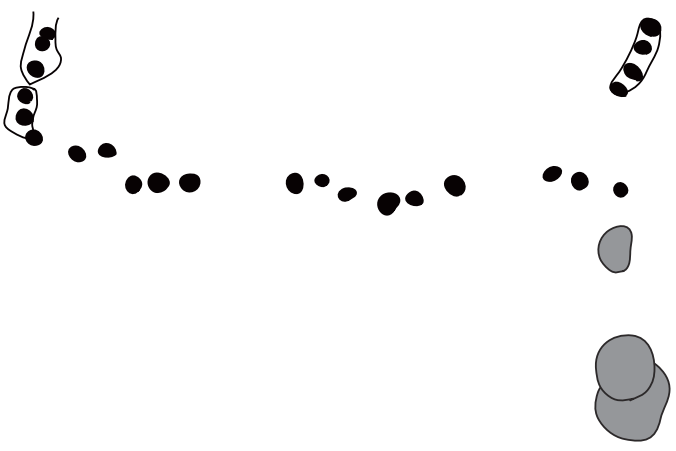

91 랄

5. La répartition des sites 
Les quelques données disponibles pour le Bronze ancien 3 vont plutôt dans le sens de cette proposition. Ainsi, en Languedoc, le site des Iragnons-Perrier à Codognan dans le Gard (Roger, Valette, 1981) illustre un habitat d'extension limitée, les vestiges se répartissant sur une surface d'environ $800 \mathrm{~m}^{2}$ [ill. 3]. Une tendance à la réduction de la taille des sites entre les phases moyenne et récente du Bronze ancien peut donc être postulée, ce qui inscrit de nouveau la France méditerranéenne dans la dynamique rhodanienne (Vital, 2008, p 186). Les fouilles du site de la Bastide Neuve II à Velaux (Bouches-du-Rhône) ont également révélé l'existence d'un petit habitat de plein air caractérisé par moins d'une dizaine de structures domestiques comprenant des témoins d'activités artisanales (Lachenal et al., 2010 ; Ropiot, Lachenal, 2011). À 500 m de là, une fouille ultérieure réalisée à la Bastide Neuve III a également livré quelques aménagements contemporains (Sargiano, 2012). Leur caractère isolé doit donc être tempéré et on peut se demander si ces deux habitats, qui partagent un même finage, participent d'une seule «agglomération » à maille très lâche ou d'un réseau de sites dispersés.

\section{Des habitats dispersés du Bronze moyen au début du Bronze final}

Pour la séquence couvrant le Bronze moyen et le début du Bronze final entre 1600 et 1350 avant notre ère (Lachenal, 2014a), qui correspond à un cycle d'évolution cohérent des sociétés protohistoriques (Vital, 1999), les données sur l'habitat ne sont pas très fournies. L'un des écueils majeurs concerne notamment la reconnaissance des structures d'habitation. En effet, seuls deux sites de cette région ont fourni des plans interprétables. Il s'agit, pour le Bronze moyen, de la structure du Moullard sud à Lambesc [ill. 4] (Boissinot et al., 1998) et pour le Bronze final 1, des deux structures accolées de Port Ariane à Lattes (Daveau, 2007). Ces habitats isolés, associés à un nombre réduit de structures de conservation, peuvent servir de modèle pour interpréter d'autres découvertes. Nombre de documents de cette époque correspondent en effet à des fosses esseulées mises au jour à l'occasion de fouilles intéressant d'autres périodes chronologiques. Certains sites rassemblent de plus nombreuses structures en creux, qui se répartissent néanmoins sur des surfaces restreintes, généralement inférieures à $1000 \mathrm{~m}^{2}$. Dans le cas des opérations les plus récentes, comme le vallon de Pié Fouquet à Rognes (Ropiot et al., 2014) ou le Petit Garlambaut à Béziers (Mazière et al., 2012), bien que les rares trous de poteaux ne permettent pas de dessiner des plans de construction, l'analyse de la densité des vestiges domestiques en position de rejet dans les fosses permet de distinguer des zones de concentration pouvant correspondre à la présence d'habitations, de l'ordre de deux à quatre au maximum. Il est ainsi possible de proposer pour cette période l'existence de modalités architecturales ne laissant que peu de traces, telles que des bâtiments à murs de terre ou reposant sur des sablières basses. Nous sommes directement confrontés ici aux limites de la reconnaissance de l'habitat en matériaux légers que de nombreux facteurs, tels que l'érosion ou la destruction des structures superficielles et des sols de circulation, contribuent à rendre invisible (Brun, 1999; Vital, 2008). La modestie des établissements du Bronze moyen et du début du Bronze final ajoute à la difficulté de les identifier, même dans le cas de diagnostics sur de grandes surfaces. Les quelques exemples disponibles indiquent l'existence d'habitats isolés pour cette période, mais il est possible d'imaginer que, comme au Bronze ancien, des établissements de ce type soient concentrés dans un même terroir. La multiplication des travaux d'archéologie préventive dans certaines villes de la vallée du Rhône, telles que Valence ou Montélimar, donne en effet des résultats allant dans ce sens (travaux de E. Néré).

En parallèle, cette période correspond à une diversification des terroirs conquis par l'habitat. Ainsi, les sites de hauteur sont de nouveau investis, en particulier dans la région des AlpesMaritimes. Certains occupent des éminences de faible extension, comme le Baou des Noirs à Vence (Vital, 1999), mais d'autres s'installent sur des sites de plus grandes dimensions, sans qu'il soit possible de déterminer la surface qu'ils occupaient, comme à la colline du Château à Nice (Lachenal, 2013). Par ailleurs, un investissement des sites littoraux installés en bordure des lagunes du golfe du Lion est particulièrement sensible.

\section{Les habitats littoraux du golfe du Lion}

Contrairement à ce qui est généralement établi, la mise en place de ces habitats littoraux remonte à la première phase du Bronze moyen (Vital, 1999). Toutes les périodes de l'âge du Bronze sont ensuite représentées, selon des proportions différentes, jusqu'à la fin du Bronze final [ill. 5]. La connaissance des formes de ces habitats lagunaires diffère en fonction des contextes. Les gisements de l'étang de l'Or sont systématiquement localisés sur de petites éminences en bordure de la lagune. Les plans des habitations n'ont pu être restitués, mais la localisation des vestiges indique une occupation de l'ensemble de la surface des plus petites buttes, ne dépassant pas les $2500 \mathrm{~m}^{2}$ (Forton, Tonnerre II), tandis que seule une partie des éminences les plus vastes sont intéressées par l'habitat (Tonnerre I : $\left.5800 \mathrm{~m}^{2}\right)$. Le gisement de Camp Redon, à Lansargue, constitue une exception, car il voit l'implantation d'un habitat de plus de 1 ha lors du Bronze final 3b (900-775/725 avant notre ère) (Dedet, Py, 1985, p. 28). Les gisements de l'étang de Thau, actuellement immergés, devaient occuper des situations topographiques similaires. Celui 
littoraux montre des zones de concentration autour des étangs de l' 0 r et de Thau. Cependant, on trouve également quelques témoins

d'occupation aux abords du complexe lagunaire de Bages-Sigean dans le

Narbonnais, à Vendres et au débouché de l'étang de Berre à Martigues.

6. Le plan du site de

Laprade à Lamotte-du-

Rhône (Vaucluse), daté du

Bronze final $2 b$, est

globalement assez aéré et

les bâtiments présentent pour la plupart la même

orientation

subméridienne, pour des

surfaces variant de 25 à

$50 \mathrm{~m}^{2}$. Des structures en creux semblent

directement associées

aux habitats, parmi

lesquels une grande fosse polylobée ayant pu servir

à l'extraction des

matériaux de

construction.

5

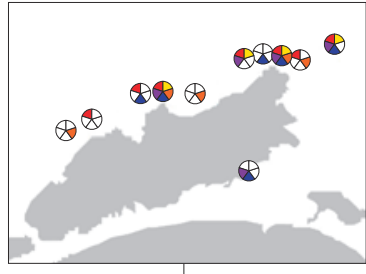

BF3

BF2

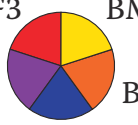

BF1
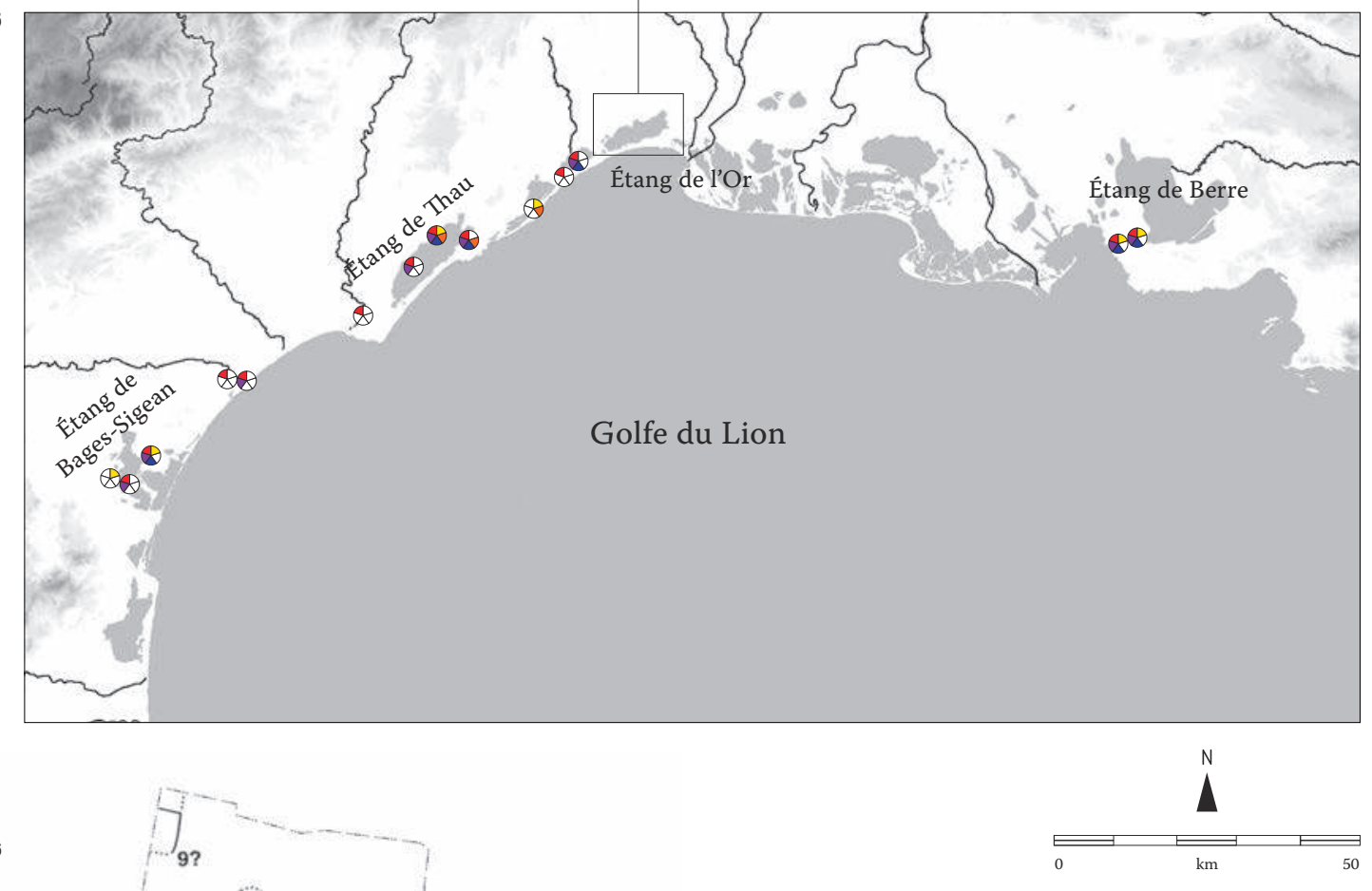

$(\because)$
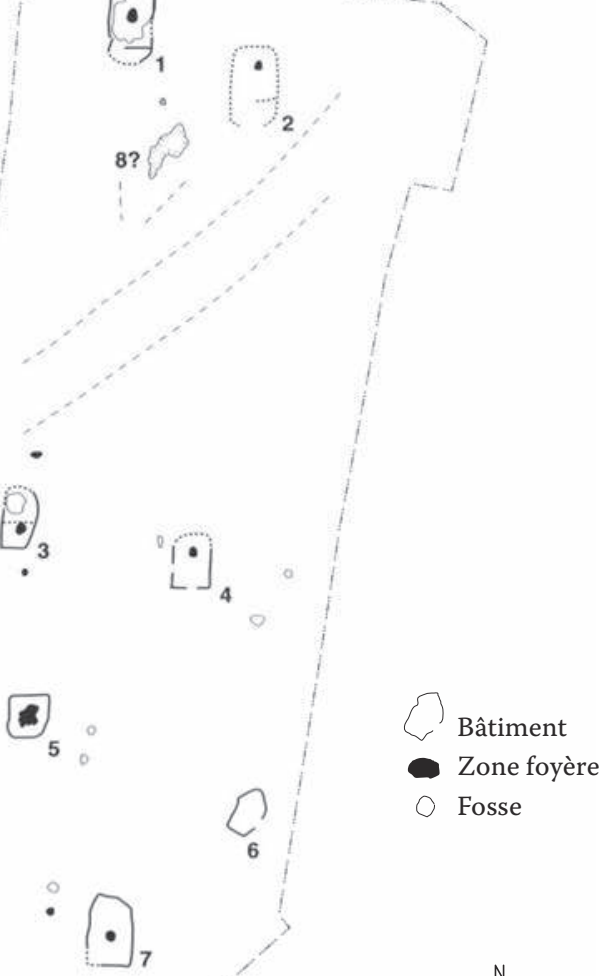

I

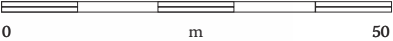


de la Fangade à Sète occupe également une petite butte. Habitée depuis la fin du Bronze moyen et jusqu'au Bronze final 3, la surface du site, matérialisé par près de 170 pieux, est estimée entre 1500 et $3000 \mathrm{~m}^{2}$ (Leroy, 2010). Sur le site de Montpenèdre à Marseillan, qui fait l'objet d'installations au Bronze final 2 et 3, l'analyse $\mathrm{du}$ plan des pieux en fonction des essences représentées permet de restituer un bâtiment rectangulaire à deux nefs de $32 \mathrm{~m}^{2}$ associées à une structure annexe et une clôture. Le gisement devait toutefois comporter d'autres habitations réparties sur une surface de $1500 \mathrm{~m}^{2}$ (ibid.). Le Roc de Conilhac à Gruissan (Aude) présente une installation originale sur une butte calcaire dominant les étangs de Bages-Sigean. Les sondages anciens (Guy, 1950) ont révélé une occupation du piémont méridional de cette éminence au Bronze moyen 1, au Bronze final $2 \mathrm{~b}$ (1150/1100-10oo avant notre ère) et 3a (1000-900 avant notre ère). Le site a en grande partie été détruit depuis, mais de nouvelles fouilles que nous avons réalisées en 2014 montrent l'extension de l'habitat sur un replat de l'éminence et sur son piémont ouest. La forte érosion subie par ce rocher ne permet toutefois pas de savoir si l'ensemble de sa surface, qui couvre un peu moins de 5 ha, était occupé à l'âge du Bronze. Les recherches récentes sur le site du Bronze final $3 b$ de la Motte à Agde, aujourd'hui immergé dans le lit du fleuve Hérault, permettent de l'interpréter comme un habitat implanté en bordure d'une lagune. Les pieux forment deux séries discontinues séparées d'environ $2 \mathrm{~m}$, organisées selon un grand axe nord-ouest/sud-est. Ils sont associés à de gros blocs de basalte dont l'agencement pose la question de structures bâties (Gascó et al., 2014). À Portal Vielh à Vendres, une fouille préventive a mis en évidence un habitat localisé en marge d'une zone humide. Le site est occupé dès le Bronze final $2 b$ et de manière continue jusqu'au Bronze final 3b. La présence d'habitations est documentée par des calages de poteaux et des fosses de stockage mais, à l'exception d'un possible grenier surélevé, aucun plan de bâtiment n'a pu être proposé. Au Bronze final 3b, le site fait l'objet d'une importante transformation de l'espace avec la réalisation d'un large fossé défensif qui enclôt une surface évaluée à 3 ha (Carozza, Burens, 200o).

À l'exception de ce dernier site, et peut-être de celui de Camp-Redon, ces établissements lagunaires ne semblent donc pas bénéficier de grandes extensions. De nombreux cas évoquent la présence d'habitats de faible densité, ou «hameaux de taille réduite» (Py, 1993, p. 50). Plusieurs sites contemporains, parfois proches les uns des autres, peuvent néanmoins être localisés autour d'un même plan d'eau, selon une densité nullement observée dans l'hinterland. Une polarisation de l'habitat autour des zones lagunaires peut donc être postulée, à l'image de ce que l'on observe pour les lacs alpins.
Le Bronze final: vers une hiérarchisation de l'habitat?

La phase moyenne du Bronze final (BF $2 b$ ), vers 1150/1100-1000 avant notre ère, présente encore de nombreux caractères communs avec les périodes antérieures. Cela se remarque en milieu lagunaire, mais également dans la persistance de sites isolés ou de petites extensions connus par quelques structures en creux (Lachenal, 2014b). La fouille du site de Laprade à Lamotte-du-Rhône est néanmoins venue enrichir ce schéma [ill. 6]. Il s'agit d'une agglomération de sept bâtiments absidiaux à architecture en terre qui, selon leur fouilleur, ne «représente qu'une faible partie d'un ensemble beaucoup plus vaste» (Billaud, 2005, p. 391). La présence de mobiliers et d'une structure de combustion contemporaine sur le site des Juilléras, à $250 \mathrm{~m}$ plus au sud (Lemercier et al., 1998 ; Lachenal, 2014b), suggère en effet que le site bénéficie d'une extension étendue, laquelle pourrait le rapprocher d'un village de manière plus concrète que pour les exemples antérieurs. La question se pose de savoir si ce site constitue une exception ou s'il s'approchait de la norme. Le caractère labile de l'architecture mis en œuvre, qui ne peut être observé qu'en cas de bonne conservation des niveaux de sols protohistoriques, permet de s'interroger sur sa présence sur d'autres sites contemporains, voire plus anciens. Toujours est-il que celle-ci se maintient ultérieurement, comme en témoigne le site du Traversant à Mailhac, daté du Bronze final 3b (Gailledrat et al., 2006-2007).

Cette phase ultime de l'âge du Bronze correspond dans le midi de la France, et en Languedoc en particulier, à une augmentation importante du nombre de découvertes (Py, 1990 ; Garcia, 2004). Celle-ci va de pair avec un accroissement du nombre de sites de hauteur, dont certains verront l'implantation d'un oppidum durant l'âge du Fer. Â côté de sites aux dimensions réduites assimilables à des habitats isolés, l'une des originalités de cette période est le développement d'établissements de plus grande dimension. Il s'agit de certains de ces sites perchés, tels que l'oppidum de Roque de Viou (Gard) ou du Cayla de Mailhac (Aude), qui atteignent chacun 6 ha (Garcia, 2004). L'ampleur réelle de leur occupation au Bronze final 3b n'est toutefois pas connue avec précision. À Mailhac, un site contemporain découvert en bas de pente au Traversant (Gailledrat et al., 2006-2007) indique la présence d'habitats dispersés ou de hameaux isolés localisés dans le territoire proche de l'oppidum, lequel fonctionne également avec la célèbre nécropole à crémation du Moulin, site éponyme du faciès languedocien du Bronze final 3b. L'oppidum du Baou-Roux (Bouches-du-Rhône) peut être comparé aux exemples languedociens. Il se démarque néanmoins d'autres habitats contemporains par la présence d'une terrasse aménagée en pierres sèches (Boissinot, 1993). Le site de Carsac à Carcassonne (Aude) occupe pour 
sa part un vaste plateau de plus de 25 ha ceinturé dès le Bronze final par un fossé muni d'une entrée en chicane. La densité de l'habitat en son centre n'est certes pas connue, mais la présence d'un fossé d'une telle dimension suggère celle d'une agglomération au statut particulier, pour laquelle l'hypothèse de lieu de marché a d'ailleurs été formulée (Guilaine et al., 1986). Dans le cas des habitats lagunaires, le site de Portal Vielh, ceinturé d'un fossé doublé d'un probable rempart de terre et de bois, fait lui aussi figure d'exception. Il pourrait également s'agir d'une agglomération d'importance, la superficie enclose étant évaluée à 3 ha. En outre, comme à Mailhac, ce site fonctionne avec une nécropole à incinération (Carozza, Burens, 200o). Le site de Camp Redon, dont la superficie s'accroît au Bronze final 3 b, pourrait également bénéficier d'un statut privilégié au sein du maillage de sites littoraux jouxtant l'étang de l'Or. Ces exemples attestent donc l'émergence d'agglomérations plus importantes à la fin du Bronze final. Ce phénomène, qui n'est pas sans rappeler le modèle de concentrationdensification proposé pour la vallée du Rhône (Vital, 2008), ne s'applique toutefois qu'à certains habitats. Il est séduisant d'y voir l'avènement d'une hiérarchisation des établissements.

\section{Des villages éclatés?}

Au terme de cette enquête, la difficulté rencontrée pour caractériser un «village» de l'âge du Bronze est confirmée en France méditerranéenne. Outre les problèmes de reconnaissance de l'habitat de cette période résultant de facteurs taphonomiques et méthodologiques, les tendances générales montrent pour toute la séquence une meilleure représentation des sites isolés et des agglomérations de petite taille. L'habitat dispersé semble donc être la modalité principale d'occupation du sol durant l'âge du Bronze, résultant d'une certaine autonomie des cellules familiales dont l'économie serait dominée par le mode de production domestique (Garcia, 2004). Deux phases se démarquent, le Bronze ancien 1 et le Bronze final $3 \mathrm{~b}$, lors desquelles on assiste à l'apparition de certains sites à surface enclose, fréquemment perchés, qui se rapprochent plus de la définition classique d'un village. Cela est d'autant plus marqué dans le cas des habitats de la fin de lâge du Bronze du Languedoc occidental qui sont associés à des nécropoles, lesquelles constituent l'une des composantes fondamentales de la définition d'un village. Ce phénomène va probablement de pair avec une hiérarchisation de l'habitat. Son identification en début et fin de période conforte le caractère non linéaire, arythmique, de l'évolution des sociétés de l'âge du Bronze.

Pour d'autres périodes, nous avons également montré que le caractère isolé des habitations ou des petits groupes de bâtiments devait être relativisé. La présence de sites contemporains dans un environnement proche permet de s'interroger sur l'existence de phénomènes de regroupements ruraux à maille très lâche, auxquels on pourrait appliquer le terme de «village éclaté » en référence au phénomène perçu au Moyen Âge dans l'ouest de la France (Pichot, 2002). Ce modèle suggère l'existence de lieux collectifs polarisant les habitats. À Saint-Maximin, le regroupement des structures de stockage pourrait remplir cette fonction. On peut également se demander si, en Languedoc occidental, les cimetières qui ne sont pas directement associés à des places centrales, interprétées comme des «nécropoles intercommunautaires » (Garcia, 2004, p. 37, 48), n'ont pas pu jouer le même rôle. Dans le cas des occupations lagunaires, bien que la concentration d'habitats soit inhérente à la recherche d'un terroir spécifique, l'existence d'un réseau organisé entre les établissements est probable, qui aboutirait à la fin de la séquence à l'émergence de sites centraux. Cette hypothèse ne saurait être appliquée à l'ensemble de l'habitat dispersé de l'âge du Bronze, elle mériterait néanmoins d'être testée dans certaines zones favorables. La multiplication des grands décapages invite à modifier notre échelle d'analyse (Brun, Marcigny, 2012) : c'est vers la reconnaissance des réseaux locaux et des systèmes de peuplement que devra s'orienter la recherche, afin de dépasser les problèmes de nomenclature et de saisir précisément le statut et l'organisation des habitats de l'âge du Bronze en France méditerranéenne. 
Audouze F., Buchsenschutz O., 1989, Villes, villages et campagnes de l'Europe celtique: du début du $\mathrm{II}^{\mathrm{e}}$ millénaire à la fin du $\mathrm{I}^{\mathrm{er}}$ siècle avant $\mathrm{J}$.-C., Paris, Hachette, 362 p.

Billaud Y., 2005, « Traces fugaces et architecture de terre au Bronze final: le cas de Laprade (Lamotte-du-Rhône, Vaucluse, TGV Méditerranée) », in Buschenschutz O. Mordant C. (DIR.), Architectures protohistoriques en Europe occidentale du Néolithique final à l'âge du Fer, Actes du $127^{\circ}$ Congrès national des Sociétés historiques et scientifiques, Nancy, 2002, Paris, CTHS, p. 389-404.

Boissinot P., 1993, Archéologie de l'habitat protohistorique. Quelques points méthodologiques (historiographie et épistémologie) examinés à partir de la fouille d'une agglomération de la périphérie massaliète, Thèse de doctorat, EHESS, $738 \mathrm{p}$.

Boissinot P., Cordier L., Marrou P., 1998, «Un transect dans le vallon du Bourrely à Lambesc, Bouches-du-Rhône (opération TGV Méditerranée).

Morphogénèse et habitat préhistorique », in D'Anna A., Binder D. (DIR.), Production et identité culturelle. Actualité de la recherche, Actes des II Rencontres méridionales de préhistoire récente, Arles, 1996, Antibes, APDCA, p. 393-401.

Brun P., 1999, «L'habitat: prisme déformant de la société protohistorique», in BRAEMER $\mathrm{F}$ Cleuziou S., Coudart A., Habitat et Société, actes des XIX' Rencontres internationales d'archéologie et d'histoire d'Antibes, Antibes, APDCA, p. 339-352.

BRUN P., MARCIGNY C., 2012, «Une connaissance de l'âge du Bronze transfigurée par l'archéologie préventive », Archéopages, H.S. 3, p. 133-139.

Carozza L., Burens A., 200o, «Les habitats du Bronze final de Portal Vielh à Vendres (Hérault) », Bulletin de la Société préhistorique française, 97, 4 , p. 573-581.

Carozza L., Marcigny C., 2007, L'Âge du Bronze en France, Paris, La Découverte, $155 \mathrm{p}$.

Cockin G., Furestier R. (DIR.), 2009, A8 SaintMaximin /Chemin de Barjols à Saint-Maximin-laSainte-Baume (Var), Rapport final d'opération. Fouilles archéologiques préventives, Mauguio, Oxford Archéol. Méditerranée, 413 p.

Coularou J., Guilaine J., Escallon G., Carrère I., 2004, "Claret, Rocher du Causse», ADLFI. Archéologie de la France - Informations. Une revue Gallia.

CourTin J., 1975, «Un habitat fortifié du Bronze ancien en Basse-Provence. Le Camp de Laure, commune du Rove (Bouches-du-Rhône) », Bulletin du Musée d'histoire naturelle de Marseille, 35, p. 217-240.

D’Anna A., Courtin J., Coutel R., Müller A., 1989, «Habitats perchés et enceintes du Néolithique final et Chalcolithique dans le Lubéron central (Vaucluse)», in D'ANNA A., GUtherz X. (DIR.), Enceintes, habitats ceinturés, sites perchés, $d u$ Néolithique au Bronze ancien dans le sud de la France et les régions voisines. Actes de la table ronde de Lattes et Aix-en-Provence, 1987, Montpellier, Éd. de la Société languedocienne de préhistoire/Adapaca, p. 166-189.

Daveau I., 2007, Port Ariane (Lattes, Hérault): construction deltaïque et utilisation d'une zone humide lors des six derniers millénaires, Lattes, Adal, 634 p.

Dedet B., Py M., 1985, L'Occupation des rivages de l'étang de Mauguio (Hérault) au Bronze final et au premier Âge du fer, t. 3, Synthèses et annexes, Caveirac, Aralo, 139 p.

Donnelly M., Furestier R. (DIR.), 2009, A8 SaintMaximin / Chemin d'Herbous à Saint-Maximinla-Sainte-Baume (Var), Rapport final d'opération. Fouilles archéologiques préventives, Mauguio, Oxford Archéologie Méditerranée, 189 p.
Gailledrat E., Boisson H., Poupet P., 2006-2007, «Le Traversan à Mailhac (Aude): un habitat de plaine du Bronze final IIIb et du Premier âge du Fer », Documents d'archéologie méridionale, 29-30, p. 19-74.

GARCia D., 2004, La Celtique méditerranéenne. Habitats et sociétés en Languedoc et en Provence du VIII ${ }^{e}$ au II siècle av. J.-C., Paris, Errance, $208 \mathrm{p}$.

Gasco J., Borja G., Tourrette C., Verdier J.-L., Bouby L., De Villers B., Greck S., Yung F., 2014, «Le site subaquatique de la Motte (Agde, Hérault) à la fin de l'âge du Bronze », in SÉNÉPART I., LÉandri F., Cauliez J., Perrin T., Thirault E. (DIR.), Chronologie de la Préhistoire récente dans le sud de la France. Acquis 1992-2012. Actualité de la recherche. Actes des $X^{e}$ Rencontres méridionales de préhistoire récente, Porticcio ( $2 A$ ), octobre 2012 , Toulouse, Archives d'écologie préhistorique, p. 625-630.

Guilaine J., Rancoule G., Vaquer J., Passelac M., VIGNE J.-D., 1986, Carsac: une agglomération protohistorique en Languedoc, Toulouse, Centre d'anthropologie des sociétés rurales, $302 \mathrm{p}$.

Guy M., 1950, «La station du Roc de Conilhac », Revue détudes ligures, 16, p. 118-125

LACHENAL T., 2013, «La céramique de l'âge du Bronze (XIV ${ }^{\mathrm{e}}$-VIII ${ }^{\mathrm{e}}$ siècles avant J.-C.) », in BouIron M. (DIR.), Nice. La colline du Château à Nice. Histoire millénaire d'une place forte, Nice, Mémoires Millénaires, p. 239-243.

LAChenal T., 2014a, «Chronologie de l'âge du Bronze en Provence», in SÉNÉPART I., LÉANDRI F. Cauliez J., Perrin T., Thirault E. (Dir.), Chronologie de la Préhistoire récente dans le sud de la France. Acquis 1992-2012. Actualité de la recherche. Actes des $X^{e}$ Rencontres méridionales de préhistoire récente, Porticcio ( $2 A)$, octobre 2012 , Toulouse, Archives d'écologie préhistorique, p. 197-220.

LACHENAL T., 2014b, «Les productions céramiques des étapes ancienne et moyenne du Bronze final en Provence ( $\mathrm{XIV}^{\mathrm{e}}-\mathrm{XI}^{\mathrm{e}}$ s. av. J.-C.) », Documents d'archéologie méridionale, 35, p. 13-51.

Lachenal T., RinalducCi De Chassey V., Georges K., Sargiano J.-P., 2010, «Une tuyère du Bronze ancien à la Bastide Neuve II (Velaux, Bouches-duRhône). Un témoin d'activité métallurgique en contexte domestique en Provence occidentale? Remarques sur les tuyères en céramique d'Europe occidentale », Bulletin de la Société préhistorique française, 107, 3, p. 549-565.

LEMERCIER O., 2002, «Les Juilléras (Mondragon) », in Buisson-Catil J., Vital J. (Dir.), Âges du Bronze en Vaucluse, Avignon, A. Barthélemy/ Département de Vaucluse, p. 61-66.

Lemercier O., Düh P., Loirat D., Mellony P., Pellissier M., Seris D., TChérémissinoff Y., BERGER J.-F., 1998, «Les Juilleras (Mondragon Vaucluse). Site d'habitat et funéraire du Néolithique récent, Néolithique final, Campaniforme - Bronze ancien et Bronze final 2b: premiers résultats », in D'ANNA A., BINDER D. (DIR.), Production et identité culturelle. Actualité de la recherche, Actes des II Rencontres méridionales de préhistoire récente, Arles, 1996 Antibes, APDCA, p. 359-368.

LEROY F., 2010, «Les habitats littoraux protohistoriques des côtes de Méditerranée nord-occidentale», in DelESTRE X., MARCHESI H. (DIR.), Archéologie des rivages méditerranéens: 50 ans de recherche, actes du colloque d'Arles, octobre 2009, Paris, Errance, p. 137-148.

LoIson G., 2003, L'Âge du Bronze ancien en Auvergne, Toulouse, EHESS (coll. Archives d'écologie préhistorique, 14), $158 \mathrm{p}$.
Mazière F., Sendra B., Convertini F., 2012, Languedoc-Roussillon, Hérault, Béziers. Le Petit Garlambaut. Un habitat du Bronze final en bordure du Libron, Rapport d'opération, Inrap Méditerranée, $239 \mathrm{p}$.

Mordant C., 2008, «L'habitat à l'âge du Bronze en France orientale», in GuILAINE J. (DIR.), Villes, villages, campagnes de l'âge du Bronze, Paris, Errance, p. 204-223.

Ріснот D., 2002, Le Village éclaté. Habitat et société dans les campagnes de l'Ouest au Moyen Âge, Rennes, Presses universitaires de Rennes, 395 p.

Py M., 1990, Culture, économie et société protohistoriques dans la région nîmoise, Rome, École française de Rome, $957 \mathrm{p}$.

Py M., 1993, Les Gaulois du Midi, de la fin de l'âge du Bronze à la conquête romaine, Hachette, Paris, $288 \mathrm{p}$

Remicourt M., Andrieu-Ponel V., Audibert C., Baradat A., Battentier J., Blaise E., Bonnardin S., Caverne J.-B., Fernandes P., Furestier R., Girard B., Lachenal T., Lepère C., Locatelli C., Martin L., Parisot N., Ponel P., Pousset D., Rué M., Schmitt A., Sénépart I, Thirault E., 2014, «Les occupations pré- et protohistoriques du Clos de Roque à SaintMaximin-la-Sainte-Baume dans le Var », in Sénépart I., Léandri F., Cauliez J., Perrin T. Thirault E. (DIr.), Chronologie de la Préhistoire récente dans le sud de la France. Acquis 1992-2012. Actualité de la recherche. Actes des $x^{e}$ Rencontres méridionales de préhistoire récente, Porticcio $(2 A)$, octobre 2012, Toulouse, Archives d'écologie préhistorique, p. 523-548.

Roger J.-M., VALETte P., 1981, «Les IragnonsPerrier,un habitat de plein air du Bronze ancien, Codognan (Gard) », Archéologie en Languedoc, 4, p. 93-102.

Ropiot V., Lachenal T., Stevenson A. (DIR.), 2014 Le Vallon de Pié Fouquet à Rognes (Bouches-duRhône), Rapport final d'opération. Fouilles archéologiques préventives, Chronoterre Archéologie, $371 \mathrm{p}$

Ropiot V., LAChenal T. (DIR.), 2011, Les Sites de la Bastide Neuve II et III. Velaux (Bouches-duRhône), Rapport final d'opération. Fouilles archéologiques préventives, Oxford Archéologie Méditerranée, 193 p.

Sargiano J.-P., 2012, Bouches-du-Rhône, Velaux, Bastide Neuve III, École départementale des sapeurs-pompiers. Occupations du début du Néolithique moyen à la Protohistoire, Rapport d'opération, Inrap Méditerranée, 195 p.

Thirault E., Charbouillot S., Durand F. Leconte C., Parisot N., Rivat F., Roux L., Rué M., Vannieuwenhuyse D., Vital J., 2013, « Petit Beaulieu à Clermont-Ferrand (Puy-de-Dôme): du Campaniforme au Bronze ancien, habitat et nécropole. Présentation préliminaire », Bulletin de l'APRAB, p. 89-93.

Vignaud A., 2002, «L'éperon du Clos Marie-Louise. Lâge du Bronze ancien », in Archéologie du TGV Méditerranée, Fiches de synthèse, t. 2, La Protohistoire, Lattes, Aralo, p. 573-576.

VITAL J., 1999, «Identification du Bronze moyen-récent en Provence et en Méditerranée nordoccidentale», Documents d'archéologie méridionale, 22, p. 7-115.

Vital J., 2008, «Architectures, sociétés, espaces durant l'âge du Bronze: quelques exemples dans le bassin rhodanien", in Guilaine J. (DIR.), Villes, villages, campagnes de l'âge du Bronze, Paris, Errance, p. 179-201.

Vital J., Convertini F., Lemercier O. (Dir.), 2012 Composantes culturelles et premières productions céramiques du Bronze ancien dans le sud-est de la France. Résultats du Projet collectif de recherche 1999-2009, Oxford, Archaeopress, 412 p.

WATtEAUX M., 2003, "À propos de la "naissance du village au Moyen Âge": la fin d'un paradigme ?», Études rurales, 167-168, 3, p. 306-318. 